\title{
On the solutions of a max-type system of difference equations with period-two parameters
}

\section{Guangwang Su*, Taixiang Sun ${ }^{1}$ and Bin Qin}

"Correspondence:
s1g6w3@163.com
${ }^{1}$ College of Information and
Statistics, Guangxi University of
Finance and Economics, Nanning,
China

\begin{abstract}
In this paper, we study the following max-type system of difference equations:

$$
\left\{\begin{array}{l}
x_{n}=\max \left\{A_{n}, \frac{y_{n-1}}{x_{n-2}}\right\}, \\
y_{n}=\max \left\{B_{n}, \frac{x_{n-1}}{y_{n-2}}\right\},
\end{array} \quad n \in\{0,1,2, \ldots\},\right.
$$

where $A_{n}, B_{n} \in(0,+\infty)$ are periodic sequences with period 2 and the initial values $x_{-1}, y_{-1}, x_{-2}, y_{-2} \in(0,+\infty)$. We show that every solution of the above system is eventually periodic.
\end{abstract}

Keywords: Max-type system of difference equations; Solution; Eventual periodicity

\section{Introduction}

Our purpose in this paper is to study eventual periodicity of the following max-type system of difference equations:

$$
\left\{\begin{array}{l}
x_{n}=\max \left\{A_{n}, \frac{y_{n-1}}{x_{n-2}}\right\}, \\
y_{n}=\max \left\{B_{n}, \frac{x_{n-1}}{y_{n-2}}\right\},
\end{array} \quad n \in \mathbf{N}_{0} \equiv\{0,1, \ldots\},\right.
$$

where $A_{n}, B_{n} \in \mathbf{R}_{+} \equiv(0,+\infty)$ are periodic sequences with period 2 and the initial values $x_{-2}, y_{-2}, x_{-1}, y_{-1} \in \mathbf{R}_{+}$.

In [1], Fotiades and Papaschinopoulos studied the following max-type system of difference equations:

$$
\left\{\begin{array}{l}
x_{n}=\max \left\{A, \frac{y_{n-1}}{x_{n-2}}\right\}, \\
y_{n}=\max \left\{B, \frac{x_{n-1}}{y_{n-2}}\right\},
\end{array} \quad n \in \mathbf{N}_{0}\right.
$$

with $A, B \in \mathbf{R}_{+}$and showed that every positive solution of (1.2) is eventually periodic.

(c) The Author(s) 2018. This article is distributed under the terms of the Creative Commons Attribution 4.0 International License (http://creativecommons.org/licenses/by/4.0/), which permits unrestricted use, distribution, and reproduction in any medium, provided you give appropriate credit to the original author(s) and the source, provide a link to the Creative Commons license, and indicate if changes were made. 
In [2], we studied the eventually periodic solutions of the following max-type system of difference equations:

$$
\left\{\begin{array}{l}
x_{n}=\max \left\{A, \frac{y_{n-k}}{x_{n-1}}\right\}, \\
y_{n}=\max \left\{B, \frac{x_{n-k}}{y_{n-1}}\right\},
\end{array} \quad n \in \mathbf{N}_{0},\right.
$$

where $A, B \in \mathbf{R}_{+}, k \in \mathbf{N} \equiv\{1,2, \ldots\}$ and the initial values $x_{-k}, y_{-k}, x_{-k+1}, y_{-k+1}, \ldots, x_{-1}, y_{-1} \in$ $\mathbf{R}_{+}$.

Recently, there has been a great interest in studying max-type systems of difference equations. In 2012, Stević in [3] obtained in an elegant way the general solution to the following max-type system of difference equations:

$$
\left\{\begin{array}{l}
x_{n+1}=\max \left\{\frac{A}{x_{n}}, \frac{y_{n}}{x_{n}}\right\}, \\
y_{n+1}=\max \left\{\frac{A}{y_{n}}, \frac{x_{n}}{y_{n}}\right\},
\end{array} \quad n \in \mathbf{N}_{0}\right.
$$

for the case $x_{0}, y_{0} \geq A>0$ and $y_{0} / x_{0} \geq \max \{A, 1 / A\}$. The solvability of various systems of difference equations has reattracted some recent interest, see, e.g., [4-6] and the references therein.

In 2016, we in [7] studied the following max-type system of difference equations:

$$
\left\{\begin{array}{l}
x_{n}=\max \left\{\frac{1}{x_{n-m}}, \min \left\{1, \frac{A}{y_{n-r}}\right\}\right\}, \\
y_{n}=\max \left\{\frac{1}{y_{n-m}}, \min \left\{1, \frac{B}{x_{n-t}}\right\}\right\},
\end{array} \quad n \in \mathbf{N}_{0},\right.
$$

where $A, B \in \mathbf{R}_{+}, m, r, t \in \mathbf{N}$ and the initial values $x_{-d}, y_{-d}, x_{-d+1}, y_{-d+1}, \ldots, x_{-1}, y_{-1} \in R_{+}$with $d=\max \{m, r, t\}$ and showed that every positive solution of (1.5) is eventually periodic with period $2 m$.

When $m=r=t=1$ and $A=B$, (1.5) reduces to the max-type system of difference equations

$$
\left\{\begin{array}{l}
x_{n}=\max \left\{\frac{1}{x_{n-1}}, \min \left\{1, \frac{A}{y_{n-1}}\right\}\right\}, \\
y_{n}=\max \left\{\frac{1}{y_{n-1}}, \min \left\{1, \frac{A}{x_{n-1}}\right\},\right.
\end{array} \quad n \in \mathbf{N}_{0} .\right.
$$

In 2015, the authors of [8] obtained the general solution of system (1.6).

Motivated by papers [9, 10], in 2014, Stević et al. in [11] investigated the following maxtype system of difference equations:

$$
\left\{\begin{array}{l}
y_{n}^{(1)}=\max _{1 \leq i_{1} \leq m_{1}}\left\{f_{1 i_{1}}\left(y_{n-k_{i_{1}, 1}^{(1)}}^{(1)}, y_{n-k_{i_{1}, 2}^{(2)}}^{(2)}, \ldots, y_{n-k_{i_{1}, l}^{(l)}}^{(l)}, n\right), y_{n-t_{1} s}^{(\sigma(1))}\right\} \\
y_{n}^{(2)}=\max _{1 \leq i_{2} \leq m_{2}}\left\{f_{2 i_{2}}\left(y_{n-k_{i_{2}, 1}^{(1)}}^{(1)}, y_{n-k_{i_{2}, 2}^{(2)}}^{(2)}, \ldots, y_{n-k_{i_{2}, l}^{(2)}}^{(l)}, n\right), y_{n-t_{2} s}^{(\sigma(2))}\right\}, \\
\ldots \\
y_{n}^{(l)}=\max _{1 \leq i_{l} \leq m_{l}}\left\{f_{l_{l} l}\left(y_{n-k_{i_{l}, 1}^{(l)}}^{(1)}, y_{n-k_{i_{l}, 2}^{(2)}}^{(2)}, \ldots, y_{n-k_{i_{l}, l}^{(l)}}^{(l)}, n\right), \mathbf{N}_{0},\right.
\end{array}\right.
$$

where $s, l, m_{j}, t_{j}, k_{i j, h}^{(j)} \in \mathbf{N}(j, h \in\{1,2, \ldots, l\}),(\sigma(1), \ldots, \sigma(l))$ is a permutation of $(1, \ldots, l)$ and $f_{j i j_{j}}: \mathbf{R}_{+}^{l} \times \mathbf{N}_{0} \longrightarrow \mathbf{R}_{+}\left(j \in\{1, \ldots, l\}\right.$ and $\left.i_{j} \in\left\{1, \ldots, m_{j}\right\}\right)$. They showed that every positive 
solution of (1.7) is eventually periodic with period $s T$ for some $T \in \mathbf{N}$ if $f_{j i}$ satisfy some conditions.

For some results of some properties of many max-type difference equations and systems, such as eventual periodicity, the boundedness character, and attractivity, see, e.g., [12-30] and the related references therein.

\section{Main results and proofs}

In this section, we study the eventual periodicity of positive solutions of system (1.1). Write $x_{2 n}=p_{n}, x_{2 n+1}=q_{n}, y_{2 n}=s_{n}, y_{2 n+1}=t_{n}$ for any $n \in \mathbf{N}_{0}$. Then system (1.1) reduces to the system

$$
\left\{\begin{array}{l}
p_{n}=\max \left\{A_{0}, \frac{t_{n-1}}{p_{n-1}}\right\}, \\
t_{n}=\max \left\{B_{1}, \frac{p_{n}}{t_{n-1}}\right\}, \quad n \in \mathbf{N}_{0}, \\
q_{n}=\max \left\{A_{1}, \frac{s_{n}}{q_{n-1}}\right\}, \\
s_{n}=\max \left\{B_{0}, \frac{q_{n-1}}{s_{n-1}}\right\},
\end{array}\right.
$$

where $A_{0}, A_{1}, B_{0}, B_{1} \in \mathbf{R}_{+}$and the initial values $s_{-1}, t_{-1}, p_{-1}, q_{-1} \in \mathbf{R}_{+}$.

The following lemma will be used in the proofs of our main results.

Lemma 2.1 Let $\left\{x_{n}\right\}_{n \geq-1}$ be a solution of the following equation:

$$
x_{n}=\max \left\{A, \frac{B}{x_{n-1}}\right\}, \quad n \in \mathbf{N}_{0}
$$

with $A, B \in \mathbf{R}_{+}$and the initial value $x_{-1} \in \mathbf{R}_{+}$. Then $x_{n}$ is eventually periodic with period 2 .

Proof By (2.2) we see $x_{n} x_{n-1} \geq B$ and $x_{n} \geq A$ for $n \in \mathbf{N}_{0}$ and for any $n \geq 2$,

$$
\begin{aligned}
A & \leq x_{n}=\max \left\{A, \frac{B}{x_{n-1}}\right\} \\
& =\max \left\{A, \frac{B x_{n-2}}{x_{n-1} x_{n-2}}\right\} \\
& \leq \max \left\{A, x_{n-2}\right\}=x_{n-2} .
\end{aligned}
$$

Then, for every $i \in\{0,1\}, x_{2 n+i}$ is eventually nonincreasing.

We claim that, for every $i \in\{0,1\}, x_{2 n+i}$ is an eventually constant sequence. Assume on the contrary that for some $i \in\{0,1\}, x_{2 n+i}$ is not an eventually constant sequence. Then there exists a sequence of positive integers $k_{1}<k_{2}<\cdots$ such that, for any $n \in \mathbf{N}$, we have

$$
\begin{gathered}
A<x_{2 k_{n+1}+i}=\frac{B}{x_{2 k_{n+1}+i-1}} \\
<x_{2 k_{n}+i}=\frac{B}{x_{2 k_{n}+i-1}},
\end{gathered}
$$

which implies $x_{2 k_{n+1}+i-1}>x_{2 k_{n}+i-1}$ for any $n \in \mathbf{N}$. This is a contradiction. Thus $x_{n}$ is eventually periodic with period 2 . The proof is complete. 
From (2.1) we see that it suffices to consider the eventual periodicity of positive solutions of the following system:

$$
\left\{\begin{array}{l}
u_{n}=\max \left\{A, \frac{v_{n-1}}{u_{n-1}}\right\}, \\
v_{n}=\max \left\{B, \frac{u_{n}}{v_{n-1}}\right\},
\end{array} \quad n \in \mathbf{N}_{0},\right.
$$

where $A, B \in \mathbf{R}_{+}$and the initial values $u_{-1}, v_{-1} \in \mathbf{R}_{+}$. Let $\left\{\left(u_{n}, v_{n}\right)\right\}_{n \geq-1}$ be a solution of (2.4). From (2.4) it immediately follows that, for any $n \in N_{0}$,

$$
u_{n} \geq A
$$

and

$$
v_{n} \geq B
$$

and

$$
\left\{\begin{array}{l}
u_{n}=\max \left\{A, \frac{B}{u_{n-1}}, \frac{1}{v_{n-2}}\right\}, \\
v_{n}=\max \left\{B, \frac{A}{v_{n-1}}, \frac{1}{u_{n-1}}\right\} .
\end{array} \quad n \in \mathbf{N},\right.
$$

Lemma 2.2 If there exist $k, p \in \mathbf{N}$ such that $u_{p+k}=u_{k}$ and $v_{p+k}=v_{k}$, then $u_{n+p}=u_{n}$ and $v_{n+p}=v_{n}$ for any $n \geq k$.

Proof It is easy to see that

$$
u_{k+p+1}=\max \left\{A, \frac{v_{k+p}}{u_{k+p}}\right\}=\max \left\{A, \frac{v_{k}}{u_{k}}\right\}=u_{k+1}
$$

and

$$
v_{k+p+1}=\max \left\{B, \frac{u_{k+p+1}}{v_{k+p}}\right\}=\max \left\{B, \frac{u_{k+1}}{v_{k}}\right\}=v_{k+1} .
$$

Assume that, for some $N \in \mathbf{N}$, we have $u_{k+p+N}=u_{k+N}$ and $v_{k+p+N}=v_{k+N}$. Then

$$
u_{k+p+N+1}=\max \left\{A, \frac{v_{k+p+N}}{u_{k+p+N}}\right\}=\max \left\{A, \frac{v_{k+N}}{u_{k+N}}\right\}=u_{k+N+1}
$$

and

$$
v_{k+p+N+1}=\max \left\{B, \frac{u_{k+p+N+1}}{v_{k+p+N}}\right\}=\max \left\{B, \frac{u_{k+N+1}}{v_{k+N}}\right\}=v_{k+N+1} .
$$

By mathematical induction, we see that $u_{n+p}=u_{n}$ and $v_{n+p}=v_{n}$ for any $n \geq k$. The proof is complete.

Proposition 2.1 If $A>B \geq 1$, then $u_{n}=A$ eventually and $v_{n}$ is eventually periodic with period 2. If $B \geq A \geq 1$, then $v_{n}=B$ eventually and $u_{n}$ is eventually periodic with period 2 . 
Proof If $A>B \geq 1$, then by (2.5)-(2.7) we see that, for $n \geq 2$,

$$
\begin{aligned}
A & \leq u_{n}=\max \left\{A, \frac{B}{u_{n-1}}, \frac{1}{v_{n-2}}\right\} \\
& \leq \max \left\{A, \frac{B}{A}, \frac{1}{B}\right\}=A .
\end{aligned}
$$

Thus, for $n \geq 2$, we have $u_{n}=A$ and

$$
v_{n}=\max \left\{B, \frac{A}{v_{n-1}}\right\} .
$$

By Lemma 2.1 we see that $v_{n}$ is eventually periodic with period 2 .

If $B \geq A \geq 1$, then by (2.5)-(2.7) we see that, for $n \geq 2$,

$$
\begin{aligned}
B & \leq v_{n}=\max \left\{B, \frac{A}{v_{n-1}}, \frac{1}{u_{n-1}}\right\} \\
& \leq \max \left\{B, \frac{A}{B}, \frac{1}{A}\right\}=B .
\end{aligned}
$$

Thus, for $n \geq 2$, we have $v_{n}=B$ and

$$
u_{n}=\max \left\{A, \frac{B}{u_{n-1}}\right\} \text {. }
$$

By Lemma 2.1 we see that $u_{n}$ is eventually periodic with period 2 . The proof is complete.

Proposition 2.2 If $B \geq 1>A \geq 1 / B$, then $v_{n}=B$ eventually and $u_{n}$ is eventually periodic with period 2 . If $1 / A>B \geq 1>A$, then $v_{n}=B$ eventually and $u_{n}$ is eventually periodic with period 2 or $u_{n}, v_{n}$ are eventually periodic with period 3.

Proof Assume that $B \geq 1>A \geq 1 / B$. By (2.5)-(2.7) we see that, for $n \geq 1$,

$$
v_{n}=\max \left\{B, \frac{A}{v_{n-1}}, \frac{1}{u_{n-1}}\right\}=B
$$

since $A / v_{n-1} \leq 1$ and $1 / u_{n-1} \leq B$. By Lemma 2.1 we see that $u_{n}$ is eventually periodic with period 2 .

Assume that $1 / A>B \geq 1>A$. Then by (2.5)-(2.7) we obtain

$$
\begin{aligned}
v_{n} & =\max \left\{B, \frac{A}{v_{n-1}}, \frac{1}{u_{n-1}}\right\} \\
& =\max \left\{B, \frac{1}{u_{n-1}}\right\} \quad(n \geq 1)
\end{aligned}
$$

since $A / v_{n-1} \leq 1$.

If $v_{n}=1 / u_{n-1}$ eventually, then $v_{n} u_{n-1}=1$ eventually and by (2.4) we have

$$
u_{n}=\max \left\{A, \frac{v_{n-1}}{u_{n-1}}\right\}=\max \left\{A, v_{n} v_{n-1}\right\}
$$




$$
=v_{n} v_{n-1} \quad \text { eventually }
$$

since $v_{n} v_{n-1} \geq B^{2}>A$. Thus from (2.9) it follows that

$$
\begin{aligned}
u_{n+3} & =v_{n+3} v_{n+2}=\frac{v_{n+3} v_{n+2} v_{n+1}}{v_{n+1}} \\
& =\frac{v_{n+3} u_{n+2} v_{n+2} v_{n+1}}{u_{n+2} v_{n+1} u_{n}} u_{n} \\
& =\frac{1 \times u_{n+2}}{u_{n+2} \times 1} u_{n}=u_{n} \quad \text { eventually, }
\end{aligned}
$$

which implies that $u_{n}, v_{n}$ are eventually periodic with period 3 .

If $v_{n}=B$ eventually, then by (2.4) we have

$$
u_{n}=\max \left\{A, \frac{B}{u_{n-1}}\right\} \quad \text { eventually. }
$$

By Lemma 2.1 we see that $u_{n}$ is eventually periodic with period 2 .

If there exists some $k \in \mathbf{N}$ such that

$$
v_{k}=B \geq \frac{1}{u_{k-1}} \quad \text { and } \quad v_{k+1}=\frac{1}{u_{k}}>B
$$

then by (2.4), (2.6), (2.8), and (2.10) it follows

$$
\begin{aligned}
u_{k+1} & =\max \left\{A, \frac{v_{k}}{u_{k}}\right\}=\max \left\{A, v_{k+1} v_{k}\right\}=v_{k+1} v_{k}, \\
v_{k+2} & =\max \left\{B, \frac{1}{u_{k+1}}\right\}=B, \\
u_{k+2} & =\max \left\{A, \frac{v_{k+1}}{u_{k+1}}\right\}=\max \left\{A, \frac{v_{k+1}}{v_{k+1} v_{k}}\right\} \\
& =\max \left\{A, \frac{1}{v_{k}}\right\}=\frac{1}{B}, \\
v_{k+3} & =\max \left\{B, \frac{1}{u_{k+2}}\right\}=B, \\
u_{k+3} & =\max \left\{A, \frac{v_{k+2}}{u_{k+2}}\right\}=B^{2}, \\
v_{k+4} & =\max \left\{B, \frac{1}{u_{k+3}}\right\}=B, \\
u_{k+4} & =\max \left\{A, \frac{v_{k+3}}{u_{k+3}}\right\}=\frac{1}{B}, \\
u_{k+5} & =\max \left\{B, \frac{1}{u_{k+4}}\right\}=B, \\
u_{k+5} & =\operatorname{sax}\left\{A, \frac{v_{k+4}}{u_{k+4}}\right\}=\frac{1}{B} .
\end{aligned}
$$


By Lemma 2.2 we see that $v_{n}=B(n \geq k+2)$ and $u_{k+2 n}=1 / B(n \geq 1)$ and $u_{k+2 n+1}=B^{2}(n \geq$ 1 ), which implies that $v_{n}=B$ eventually and $u_{n}$ is eventually periodic with period 2 . The proof is complete.

Proposition 2.3 If $A \geq 1>B$, then $u_{n}=A$ eventually and $v_{n}$ is eventually periodic with period 2.

Proof If $A \geq 1>B \geq 1 / A$, then by (2.5)-(2.7) we see that, for $n \geq 2$,

$$
u_{n}=\max \left\{A, \frac{B}{u_{n-1}}, \frac{1}{v_{n-2}}\right\}=A
$$

since $1 / v_{n-2} \leq A$ and $B<u_{n-1}$. Thus from (2.4) it follows

$$
v_{n}=\max \left\{B, \frac{A}{v_{n-1}}\right\} \quad \text { eventually. }
$$

By Lemma 2.1 we see that $v_{n}$ is eventually periodic with period 2 .

Now assume that $1 / B>A \geq 1>B$. We claim that there exists a sequence of positive integers $n_{1}<n_{2}<\cdots$ such that $u_{n_{k}}=A$. Indeed, if $u_{n}=v_{n-1} / u_{n-1}>A$ eventually, then

$$
\begin{aligned}
A^{2} & <u_{n} u_{n-1}=v_{n-1}=\max \left\{B, \frac{u_{n-1}}{v_{n-2}}\right\} \\
& =\frac{u_{n-1}}{v_{n-2}}=\max \left\{\frac{A}{v_{n-2}}, \frac{1}{u_{n-2}}\right\} \\
& =\frac{1}{u_{n-2}} \text { eventually, }
\end{aligned}
$$

which implies $1 \leq A^{3}<u_{n} u_{n-1} u_{n-2}=1$, a contradiction.

If $u_{n}=A$ eventually, then by Lemma 2.1 we see that $v_{n}$ is eventually periodic with pe$\operatorname{riod} 2$.

If there exists some $k \in \mathbf{N}$ such that

$$
u_{k}=A \geq \frac{v_{k-1}}{u_{k-1}} \quad \text { and } \quad u_{k+1}=\frac{v_{k}}{u_{k}}=\frac{v_{k}}{A}>A
$$

then $v_{k}=u_{k+1} u_{k}>A^{2}$ and by (2.4) and (2.11) it follows

$$
\begin{aligned}
& v_{k+1}=\max \left\{B, \frac{u_{k+1}}{v_{k}}\right\}=\max \left\{B, \frac{1}{u_{k}}\right\}=\frac{1}{A}, \\
& u_{k+2}=\max \left\{A, \frac{v_{k+1}}{u_{k+1}}\right\}=\max \left\{A, \frac{1}{v_{k}}\right\}=A, \\
& v_{k+2}=\max \left\{B, \frac{u_{k+2}}{v_{k+1}}\right\}=A^{2}, \\
& u_{k+3}=\max \left\{A, \frac{v_{k+2}}{u_{k+2}}\right\}=A, \\
& v_{k+3}=\max \left\{B, \frac{u_{k+3}}{v_{k+2}}\right\}=\frac{1}{A},
\end{aligned}
$$




$$
\begin{aligned}
& u_{k+4}=\max \left\{A, \frac{v_{k+3}}{u_{k+3}}\right\}=A, \\
& v_{k+4}=\max \left\{B, \frac{u_{k+4}}{v_{k+3}}\right\}=A^{2} .
\end{aligned}
$$

By Lemma 2.2 we see that $u_{n}=A(n \geq k+2)$ and $v_{k+2 n-1}=1 / A(n \geq 1)$ and $v_{k+2 n}=A^{2}(n \geq$ 1 ), which implies that $u_{n}=A$ eventually and $v_{n}$ is eventually periodic with period 2 . The proof is complete.

Proposition 2.4 If $A<1$ and $B<1$, then $u_{n}=A$ eventually and $v_{n}$ is eventually periodic with period 2 or $v_{n}=B$ eventually and $u_{n}$ is eventually periodic with period 2 or $u_{n}, v_{n}$ are eventually periodic with period 3.

Proof Note

$$
u_{n}=\max \left\{A, \frac{v_{n-1}}{u_{n-1}}\right\} \text {. }
$$

There are three cases to consider.

Case 1. Assume that $u_{n}=A$. By Lemma 2.1 we see that $v_{n}$ is eventually periodic with period 2 .

Case 2. Assume that

$$
u_{n}=v_{n-1} / u_{n-1}>A \quad \text { eventually }
$$

Then by (2.7) it follows

$$
v_{n}=\max \left\{B, \frac{A}{v_{n-1}}, \frac{1}{u_{n-1}}\right\}=\max \left\{B, \frac{1}{u_{n-1}}\right\} \text { eventually. }
$$

If $v_{n}=B$ eventually, then by Lemma 2.1 we see that $u_{n}$ is eventually periodic with period 2 .

If $v_{n}=1 / u_{n-1}>B$ eventually, then by $(2.12)$ we have

$$
\begin{aligned}
u_{n+3} & =\frac{v_{n+2}}{u_{n+2}}=\frac{1}{u_{n+2} u_{n+1}} \\
& =\frac{u_{n}}{u_{n+2} u_{n+1} u_{n}} \\
& =\frac{u_{n}}{v_{n+1} u_{n}} \\
& =u_{n} \quad \text { eventually, }
\end{aligned}
$$

which implies that $u_{n}, v_{n}$ are eventually periodic with period 3 .

In the following, we assume that there exists some $k \in \mathbf{N}$ such that, for every $n \geq k$,

$$
u_{n}=\frac{v_{n-1}}{u_{n-1}}, \quad v_{k}=B, \quad v_{k+1}=\frac{1}{u_{k}}>B .
$$


Thus by (2.13) and (2.14) it follows

$$
\begin{aligned}
u_{k+1} & =\frac{B}{u_{k}} \\
u_{k+2} & =\frac{v_{k+1}}{u_{k+1}}=\frac{1}{B}, \\
v_{k+2} & =\max \left\{B, \frac{1}{u_{k+1}}\right\}=\max \left\{B, \frac{u_{k}}{B}\right\} .
\end{aligned}
$$

If $v_{k+2}=B \geq u_{k} / B$, then by (2.13)-(2.16) we have

$$
\begin{aligned}
& u_{k+3}=\frac{v_{k+2}}{u_{k+2}}=B^{2}, \\
& v_{k+3}=\max \left\{B, \frac{1}{u_{k+2}}\right\}=B, \\
& u_{k+4}=\frac{v_{k+3}}{u_{k+3}}=\frac{1}{B}, \\
& v_{k+4}=\max \left\{B, \frac{1}{u_{k+3}}\right\}=\frac{1}{B^{2}}, \\
& u_{k+5}=\frac{v_{k+4}}{u_{k+4}}=\frac{1}{B}, \\
& v_{k+5}=\max \left\{B, \frac{1}{u_{k+4}}\right\}=B .
\end{aligned}
$$

By Lemma 2.2 we see that $u_{n+3}=u_{n}$ and $v_{n+3}=v_{n}(n \geq k+2)$, which implies that $u_{n}, v_{n}$ are eventually periodic with period 3 .

If $v_{k+2}=u_{k} / B>B$, then by $(2.13)-(2.16)$ we have

$$
\begin{aligned}
& u_{k+3}=\frac{v_{k+2}}{u_{k+2}}=u_{k}, \\
& v_{k+3}=\max \left\{B, \frac{1}{u_{k+2}}\right\}=B, \\
& u_{k+4}=\frac{v_{k+3}}{u_{k+3}}=\frac{B}{u_{k}}, \\
& v_{k+4}=\max \left\{B, \frac{1}{u_{k+3}}\right\}=\frac{1}{u_{k}}, \\
& u_{k+5}=\frac{v_{k+4}}{u_{k+4}}=\frac{1}{B}, \\
& v_{k+5}=\max \left\{B, \frac{1}{u_{k+4}}\right\}=\frac{u_{k}}{B} .
\end{aligned}
$$

By Lemma 2.2 we see that $u_{n+3}=u_{n}$ and $v_{n+3}=v_{n}(n \geq k+2)$, which also implies that $u_{n}, v_{n}$ are eventually periodic with period 3 .

Case 3. Assume that there exists some $k \in \mathbf{N}$ such that

$$
u_{k}=A \geq \frac{v_{k-1}}{u_{k-1}}, \quad u_{k+1}=\frac{v_{k}}{u_{k}}=\frac{v_{k}}{A}>A .
$$


Then $v_{k}=u_{k+1} u_{k}>A^{2}$ and by (2.7) and (2.17) we have

$$
v_{k+1}=\max \left\{B, \frac{A}{v_{k}}, \frac{1}{u_{k}}\right\}=\max \left\{B, \frac{1}{A}\right\}=\frac{1}{A}
$$

and

$$
u_{k+2}=\max \left\{A, \frac{v_{k+1}}{u_{k+1}}\right\}=\max \left\{A, \frac{1}{v_{k}}\right\} .
$$

If $u_{k+2}=A \geq 1 / v_{k}$ and $\sqrt{B} \geq A \geq B^{2}$, then by (2.4), (2.18), and (2.19) it follows

$$
\begin{aligned}
& v_{k+2}=\max \left\{B, \frac{u_{k+2}}{v_{k+1}}\right\}=B, \\
& u_{k+3}=\max \left\{A, \frac{v_{k+2}}{u_{k+2}}\right\}=\frac{B}{A}, \\
& v_{k+3}=\max \left\{B, \frac{u_{k+3}}{v_{k+2}}\right\}=\frac{1}{A}, \\
& u_{k+4}=\max \left\{A, \frac{v_{k+3}}{u_{k+3}}\right\}=\frac{1}{B}, \\
& v_{k+4}=\max \left\{B, \frac{u_{k+4}}{v_{k+3}}\right\}=\frac{A}{B}, \\
& u_{k+5}=\max \left\{A, \frac{v_{k+4}}{u_{k+4}}\right\}=A, \\
& v_{k+5}=\max \left\{B, \frac{u_{k+5}}{v_{k+4}}\right\}=B .
\end{aligned}
$$

By Lemma 2.2 we see that $u_{n+3}=u_{n}$ and $v_{n+3}=v_{n}(n \geq k+2)$, which implies that $u_{n}, v_{n}$ are eventually periodic with period 3 .

If $u_{k+2}=A \geq 1 / v_{k}$ and $\sqrt{B}>B^{2}>A$, then by (2.4), (2.18), and (2.19) it follows

$$
\begin{aligned}
& v_{k+2}=B, \quad u_{k+3}=\frac{B}{A}, \\
& v_{k+3}=\frac{1}{A}, \quad u_{k+4}=\frac{1}{B}, \\
& v_{k+4}=\max \left\{B, \frac{u_{k+4}}{v_{k+3}}\right\}=B, \\
& u_{k+5}=\max \left\{A, \frac{v_{k+4}}{u_{k+4}}\right\}=B^{2}, \\
& v_{k+5}=\max \left\{B, \frac{u_{k+5}}{v_{k+4}}\right\}=B, \\
& u_{k+6}=\max \left\{A, \frac{v_{k+5}}{u_{k+5}}\right\}=\frac{1}{B}, \\
& v_{k+6}=\max \left\{B, \frac{u_{k+6}}{v_{k+5}}\right\}=\frac{1}{B^{2}}, \\
& u_{k+7}=\max \left\{A, \frac{v_{k+6}}{u_{k+6}}\right\}=\frac{1}{B},
\end{aligned}
$$




$$
v_{k+7}=\max \left\{B, \frac{u_{k+7}}{v_{k+6}}\right\}=B
$$

By Lemma 2.2 we see that $u_{n+3}=u_{n}$ and $v_{n+3}=v_{n}(n \geq k+4)$, which implies that $u_{n}, v_{n}$ are eventually periodic with period 3 .

If $u_{k+2}=A \geq 1 / v_{k}$ and $\sqrt{B}<A$, then by (2.4), (2.18), and (2.19) it follows

$$
\begin{aligned}
& v_{k+2}=\max \left\{B, \frac{u_{k+2}}{v_{k+1}}\right\}=A^{2}, \\
& u_{k+3}=\max \left\{A, \frac{v_{k+2}}{u_{k+2}}\right\}=A, \\
& v_{k+3}=\max \left\{B, \frac{u_{k+3}}{v_{k+2}}\right\}=\frac{1}{A}, \\
& u_{k+4}=\max \left\{A, \frac{v_{k+3}}{u_{k+3}}\right\}=\frac{1}{A^{2}}, \\
& v_{k+4}=\max \left\{B, \frac{u_{k+4}}{v_{k+3}}\right\}=\frac{1}{A}, \\
& u_{k+5}=\max \left\{A, \frac{v_{k+4}}{u_{k+4}}\right\}=A, \\
& v_{k+5}=\max \left\{B, \frac{u_{k+5}}{v_{k+4}}\right\}=A^{2} .
\end{aligned}
$$

By Lemma 2.2 we see that $u_{n+3}=u_{n}$ and $v_{n+3}=v_{n}(n \geq k+2)$, which implies that $u_{n}, v_{n}$ are eventually periodic with period 3 .

If $u_{k+2}=1 / v_{k}>A \geq B v_{k}$, then by (2.4), (2.18), and (2.19) it follows

$$
\begin{aligned}
& v_{k+2}=\max \left\{B, \frac{u_{k+2}}{v_{k+1}}\right\}=\frac{A}{v_{k}}, \\
& u_{k+3}=\max \left\{A, \frac{v_{k+2}}{u_{k+2}}\right\}=A, \\
& v_{k+3}=\max \left\{B, \frac{u_{k+3}}{v_{k+2}}\right\}=v_{k}, \\
& u_{k+4}=\max \left\{A, \frac{v_{k+3}}{u_{k+3}}\right\}=\frac{v_{k}}{A}, \\
& v_{k+4}=\max \left\{B, \frac{u_{k+4}}{v_{k+3}}\right\}=\frac{1}{A}, \\
& u_{k+5}=\max \left\{A, \frac{v_{k+4}}{u_{k+4}}\right\}=\frac{1}{v_{k}}, \\
& v_{k+5}=\max \left\{B, \frac{u_{k+5}}{v_{k+4}}\right\}=\frac{A}{v_{k}} .
\end{aligned}
$$

By Lemma 2.2 we see that $u_{n+3}=u_{n}$ and $v_{n+3}=v_{n}(n \geq k+2)$, which implies that $u_{n}, v_{n}$ are eventually periodic with period 3 .

If $u_{k+2}=1 / v_{k}>A$ and $A / B<v_{k} \leq 1 / B^{2}$, then by (2.4), (2.18), and (2.19) it follows

$$
v_{k+2}=\max \left\{B, \frac{u_{k+2}}{v_{k+1}}\right\}=B,
$$




$$
\begin{aligned}
& u_{k+3}=\max \left\{A, \frac{v_{k+2}}{u_{k+2}}\right\}=B v_{k}, \\
& v_{k+3}=\max \left\{B, \frac{u_{k+3}}{v_{k+2}}\right\}=v_{k}, \\
& u_{k+4}=\max \left\{A, \frac{v_{k+3}}{u_{k+3}}\right\}=\frac{1}{B}, \\
& v_{k+4}=\max \left\{B, \frac{u_{k+4}}{v_{k+3}}\right\}=\max \left\{B, \frac{1}{B v_{k}}\right\}=\frac{1}{B v_{k}}, \\
& u_{k+5}=\max \left\{A, \frac{v_{k+4}}{u_{k+4}}\right\}=\frac{1}{v_{k}}, \\
& v_{k+5}=\max \left\{B, \frac{u_{k+5}}{v_{k+4}}\right\}=B .
\end{aligned}
$$

By Lemma 2.2 we see that $u_{n+3}=u_{n}$ and $v_{n+3}=v_{n}(n \geq k+2)$, which implies that $u_{n}, v_{n}$ are eventually periodic with period 3 .

If $u_{k+2}=1 / v_{k}>A$ and $v_{k}>1 / B^{2}$, then $A<B^{2}$ and by (2.4), (2.18), and (2.19) it follows

$$
\begin{aligned}
& v_{k+2}=B, \quad u_{k+3}=B v_{k}, \\
& v_{k+3}=v_{k}, \quad u_{k+4}=\frac{1}{B}, \\
& v_{k+4}=\max \left\{B, \frac{u_{k+4}}{v_{k+3}}\right\}=\max \left\{B, \frac{1}{B v_{k}}\right\}=B, \\
& u_{k+5}=\max \left\{A, \frac{v_{k+4}}{u_{k+4}}\right\}=B^{2}, \\
& v_{k+5}=\max \left\{B, \frac{u_{k+5}}{v_{k+4}}\right\}=B, \\
& u_{k+6}=\max \left\{A, \frac{v_{k+5}}{u_{k+5}}\right\}=\frac{1}{B}, \\
& v_{k+6}=\max \left\{B, \frac{u_{k+6}}{v_{k+5}}\right\}=\frac{1}{B^{2}}, \\
& u_{k+7}=\max \left\{A, \frac{v_{k+6}}{u_{k+6}}\right\}=\frac{1}{B}, \\
& v_{k+7}=\max \left\{B, \frac{u_{k+7}}{v_{k+6}}\right\}=B .
\end{aligned}
$$

By Lemma 2.2 we see that $u_{n+3}=u_{n}$ and $v_{n+3}=v_{n}(n \geq k+4)$, which implies that $u_{n}, v_{n}$ are eventually periodic with period 3 . The proof is complete.

Combining (2.1) with (2.3), from Propositions 2.1-2.4 we obtain the following theorem.

Theorem 2.1 Let $\left\{\left(x_{n}, y_{n}\right)\right\}_{n \geq-2}$ be a positive solution of (1.1). Then $x_{n}$ and $y_{n}$ are eventually periodic with periods $T_{x}$ and $T_{y}$, respectively, and $T_{x}, T_{y} \in\{2,4,6,12\}$. 


\section{Funding}

The research was supported by NNSF of China (11761011) and NSF of Guangxi (2016GXNSFBA380235,

2016GXNSFAA380286) and YMTBAPP of Guangxi Colleges (2017KY0598) and PIT of Guangxi University of Finance and Economics.

\section{Competing interests}

The authors declare that they have no competing interests.

\section{Authors' contributions}

All authors participated in every phase of research conducted for this paper. All authors read and approved the final manuscript.

\section{Publisher's Note}

Springer Nature remains neutral with regard to jurisdictional claims in published maps and institutional affiliations.

\section{Received: 10 September 2018 Accepted: 30 September 2018 Published online: 10 October 2018}

\section{References}

1. Fotiades, E., Papaschinopoulos, G.: On a system of difference equations with maximum. Appl. Math. Comput. 221, 684-690 (2013)

2. Su, G., Sun, T., Qin, B.: Eventually periodic solutions of a max-type system of difference equations of higher order. Discrete Dyn. Nat. Soc. 2018, Article ID 8467682 (2018)

3. Stević, S.: Solutions of a max-type system of difference equations. Appl. Math. Comput. 218, 9825-9830 (2012)

4. Stević, S., Diblik, J., Iričanin, D.B., Smarda, Z.: On some solvable difference equations and systems of difference equations. Abstr. Appl. Anal. 2012, Article ID 541761 (2012)

5. Stević, S., Iričanin, D.B., Smarda, Z:: On a product-type system of difference equations of second order solvable in closed form. J. Inequal. Appl. 2015, 327 (2015)

6. Stević, S., Iričanin, D.B., Smarda, Z.: Two-dimensional product-type system of difference equations solvable in closed form. Adv. Differ. Equ. 2016, 253 (2016)

7. Sun, T., Xi, H.: On the solutions of a system of difference equations with maximum. Appl. Math. Comput. 290, 292-297 (2016)

8. Yazlik, Y., Tollu, T.D., Taskara, N.: On the solutions of a max-type difference equation system. Math. Methods Appl. Sci. 38, 4388-4410 (2015)

9. Stević, S.: Periodicity of max difference equations. Util. Math. 83, 69-71 (2010)

10. Stević, S.: On some periodic systems of max-type difference equations. Appl. Math. Comput. 218, 11483-11487 (2012)

11. Stević, S., Alghamdi, A.M., Alotaibi, A., Shahzad, N.: Eventual periodicity of some systems of max-type difference equations. Appl. Math. Comput. 236, 635-641 (2014)

12. Berenhaut, S.K., Foley, D.J., Stević, S.: Boundedness character of positive solutions of a max difference equation. J. Differ. Equ. Appl. 12, 1193-1199 (2006)

13. Elsayed, M.E., Iričanin, D.B., Stević, S.: On the max-type equation $x_{n+1}=\max \left\{A_{n} / x_{n}, x_{n-1}\right\}$. Ars Comb. 95, 187-192 (2010)

14. Liu, W., Yang, X., Stević, S.: On a class of nonautonomous max-type difference equations. Abstr. Appl. Anal. 2011, Article ID 327432 (2011)

15. Qin, B., Sun, T., Xi, H.: Dynamics of the max-type difference equation $x_{n+1}=\max \left\{A / x_{n}, x_{n-k}\right\}$. J. Comput. Anal. Appl. 14, 856-861 (2012)

16. Sauer, T.: Global convergence of max-type equations. J. Differ. Equ. Appl. 17, 1-8 (2011)

17. Shi, Q., Su, X., Yuan, G.: Characters of the solutions to a generalized nonlinear max-type difference equation. Chin. Ann. Math., Ser. B 28, 284-289 (2013)

18. Stević, S.: Global stability of a max-type difference equation. Appl. Math. Comput. 216, 354-356 (2010)

19. Stević, S.: On a generalized max-type difference equation from automatic control theory. Nonlinear Anal., Theory Methods Appl. 72, 1841-1849 (2010)

20. Stević, S.: On a nonlinear generalized max-type difference equation. J. Math. Anal. Appl. 376, 317-328 (2011)

21. Stević, S.: On a symmetric system of max-type difference equations. Appl. Math. Comput. 219, 8407-8412 (2013)

22. Stević, S.: Product-type system of difference equations of second-order solvable in closed form. Electron. J. Qual. Theory Differ. Equ. 56, 1 (2015)

23. Stević, S.: Representation of solutions of bilinear difference equations in terms of generalized Fibonacci sequences. Electron. J. Qual. Theory Differ. Equ. 67, 1 (2014)

24. Stević, S., Alghamdi, A.M., Alotaibi, A., Shahzad, N.: Boundedness character of a max-type system of difference equations of second order. Electron. J. Qual. Theory Differ. Equ. 45, 1 (2014)

25. Sun, T., He, Q., Wu, X., Xi, H.: Global behavior of the max-type difference equation $x_{n}=\max \left\{1 / x_{n-m}, A_{n} / x_{n-1}\right\}$. Appl. Math. Comput. 248, 687-692 (2014)

26. Sun, T., Liu, J., He, Q., Liu, X.: Eventually periodic solutions of a max-type difference equation. Sci. World J. 2014, Article ID $219437(2014)$

27. Sun, T., Qin, B., Xi, H., Han, C.: Global behavior of the max-type difference equation $x_{n+1}=\max \left\{1 / x_{n}, A_{n} / x_{n-1}\right\}$. Abstr. Appl. Anal. 2009, Article ID 152964 (2009)

28. Sun, T., Xi, H., Han, C., Qin, B.: Dynamics of the max-type difference equation $x_{n}=\max \left\{1 / x_{n-m}, A_{n} / x_{n-r}\right\}$. J. Appl. Math. Comput. 38, 173-180 (2012)

29. Sun, T., Xi, H., Quan, W.: Existence of monotone solutions of a difference equation. Discrete Dyn. Nat. Soc. 2008, Article ID 917560 (2008)

30. Xiao, Q., Shi, Q.: Eventually periodic solutions of a max-type equation. Math. Comput. Model. 57, 992-996 (2013) 